\title{
Information flows and processes in a SME network
}

\author{
F. Bonfatti, P. D. Monari, R. Montanari \\ Department of Engineering Sciences - University of Modena \\ Via Campi 213/B - 41100 Modena (Italy) \\ tel +3959 378514, fax +3959378515 \\ e-mailbonfatti@unimo.it
}

\begin{abstract}
The paper presents and discusses the main informational activities that take place in a network of small-medium manufacturing enterprises. The network management process is split into elementary steps, grouped according to the entity in charge of them: the network coordinating unit or the single node. The identified steps are those that ensure high reactivity of the network as a whole to customer requests, and the required autonomy and equal rights to the network nodes. The states of the network result strongly related to those of two basic informational elements: the customer order and the node task. The analysis has been carried out to provide requirements for the software tools that should support the network activities.
\end{abstract}

Keywords

Virtual enterprise, SME, information flow, information management, Esprit project

\section{INTRODUCTION}

In recent years the concepts of holonic system (Koestler, 1989), virtual enterprise (Rolstadas, 1994), distributed manufacturing environment (Hirsch, 1995) and similar are gaining increasing importance and diffusion. A promising type of virtual enterprise is that obtained by organizing small-medium sized enterprises (SMEs) into a coordinated network (Bonfatti, 1995). The benefits that come to the single SME from joining a network include access to new business opportunites, knowledge exchange, costs and risks sharing, improvement of specialization.

Enterprise network management is the subject studied by the EP project 20723 - PLENT (Planning Small-Medium Enterprise Networks) of the European ESPRIT programme. The project is aimed at designing and developing a set of software tools (PLENT, 1997) to support the activities of a SME network in terms of:

- workload distribution among nodes to satisfy customer orders, 
- reactive process planning at the nodes to meet the network manufacturing objectives,

- evaluation of node performances to ascertain their reliability.

According to the PLENT approach, the network organization relies on three basic principles (PLENT, 1996).

- The network is a stable structure. It means that only a regimen behaviours are studied, while transient events such as network constitution or modification are not considered.

- The nodes have ensured equal rights. All the nodes are potentially enabled to perform the same functions, although they differ for the tasks they can execute.

- Each node maintains its complete autonomy. Modification of the current organization, including its information system and procedures, is not required.

As a matter of fact, SMEs present positive qualities to behave like network nodes thanks to their lean structure, adaptability to market evolution, active involvement of versatile human resources, habit to establish subcontracting relations, good technological level of their products. But a major obstacle to coordination is the traditional individualistic attitude of SMEs, the same attitude that, in other times, gave them the motivation for starting and growing their business. This attitude leaves each enterprise completely alone in facing marketing, purchasing, design, engineering and technological innovation problems. In addition, other problems arise from historical distrusts between enterprises traditionally in competition with each other.

This situation cannot be overcome by a centralized planning system, acting on behalf of the single nodes. It would result in overlapping the local systems and subtracting node responsibilities, thus introducing unbearable interferences with the single node policy. In addition, this solution would require a centralized detailed knowledge of production plan progress of all the nodes and, consequently, a burdensome information exchange.

For opposite reasons, a system relying on spontaneous self-regulation mechanisms, such as those proposed for large enterprises that decentralize their business, would keep open the distrust problems cited above. This kind of network is based on horizontal and vertical communications between nodes that do not ensure a global system vision and do not remove the current interdependencies between enterprises (where the largest or most powerful enterprise, or that supplying a critical component, determines the supply policy).

It is the aim of the PLENT project to propose a SME network organization that ensures fast response to costumer request while keeping the required independence degree of the node enterprises in due consideration. This approach is based on the neutral application of a number of clear, objective rules, agreed upon by all the network nodes, concerning task distribution and node reliability evaluation. In other terms, the key aspects are two: coordinated planning and node behaviour measurement. The choice of the most suitable decision-making and optimization algorithms comes later and is, in some sense, less important.

The management of this type of network considers the roles of three fundamental entities, customer, coordinating unit and nodes, and the interactions between them (Bonfatti, 1996).

\section{Customer (C)}

Customers represent the external entities which activate the network processes. All the communications between the customer and the network are managed by the coordinating unit, 
hence the customer does not see nodes, with the only exception of that delivering the final product.

\section{Coordinating unit $(C U)$}

The CU main function is to collect external orders and decide how to involve the network nodes in the manufacturing process. To this aim, it must keep an updated view of the current nodes state and reliability, and it is directly informed by nodes whenever problems arise. Finally, the CU has to manage all the information which is necessary to evaluate network nodes performances.

\section{Nodes $\left(N_{i}\right)$}

Their duty is to carry out the tasks which are assigned to them by the $\mathrm{CU}$, so as to deliver to the next node (or to the customer, if the node is the last one) the right quantity of product within the due date. Moreover, every node has to inform the $\mathrm{CU}$ about problems causing delays or wastes of material. Short and medium term planning of manufacturing activities are basic steps of node behaviour.

The modalities of interaction of these entities, the information they exchange, the processes they execute in normal and emergency conditions, constitute basic requirements for software design. Hence, the first phase of the PLENT project has been devoted to a detailed study of these aspects. The paper reports the results of this study. Section 2 examines the states assumed by the two main informational elements: customer order and node task. Then, Sections 3 and 4 present, by means of data flow diagrams, the processes that take place within the single node and the coordinating unit, respectively, as well as the information they exchange.

\section{ORDERS AND TASKS}

The network external demand is represented by customer orders addressed to the CU, requiring a specific type product. Each order is defined by a triplet $\langle p, q, d d\rangle$ (product type, ordered quantity, due date). The states that the order can take during its processing are reported in the state diagram of Figure 1.

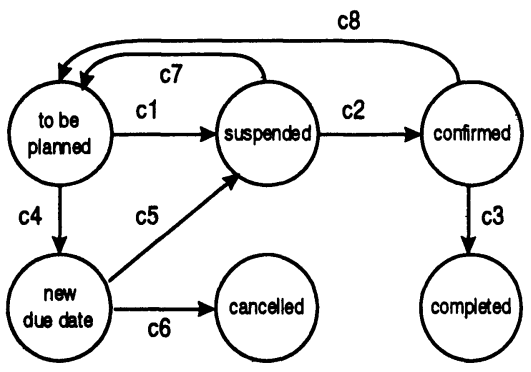

Figure 1 State diagram of the customer order. c1: positive planning phase evaluation c2: nodes availability c3: end of the last task c4: negative planning phase evaluation C5: alternative due date accoptance c6: alternative due date rejection c7: not available node (rejection message) c8: delay detection (delay message) 
Each time a customer sends a request to the $\mathrm{CU}$, a new order instance is created whose initial state is to be planned. Then, the assignment process decomposing the order into $n$ tasks is executed, and the order assumes the suspended state. In fact, only when all the nodes involved in tasks distribution confirm their availability, the order state becomes confirmed. Finally, after all the manufacturing steps have been accomplished, the order changes its state into completed.

If the $\mathrm{CU}$ is not able to determine an executable task distribution, the order passes from the to be planned state to the new due date state. Starting from this state, and according to the customer decision, the order can assume the to be planned state value again or be cancelled.

Transitions $c 7$ and $c 8$ occur whenever a new planning phase is required. This case occurs when a node rejects the assigned task or introduces delays. Replanning activities can determine new task distributions (c1) or the suggestion of alternative due dates (c4).

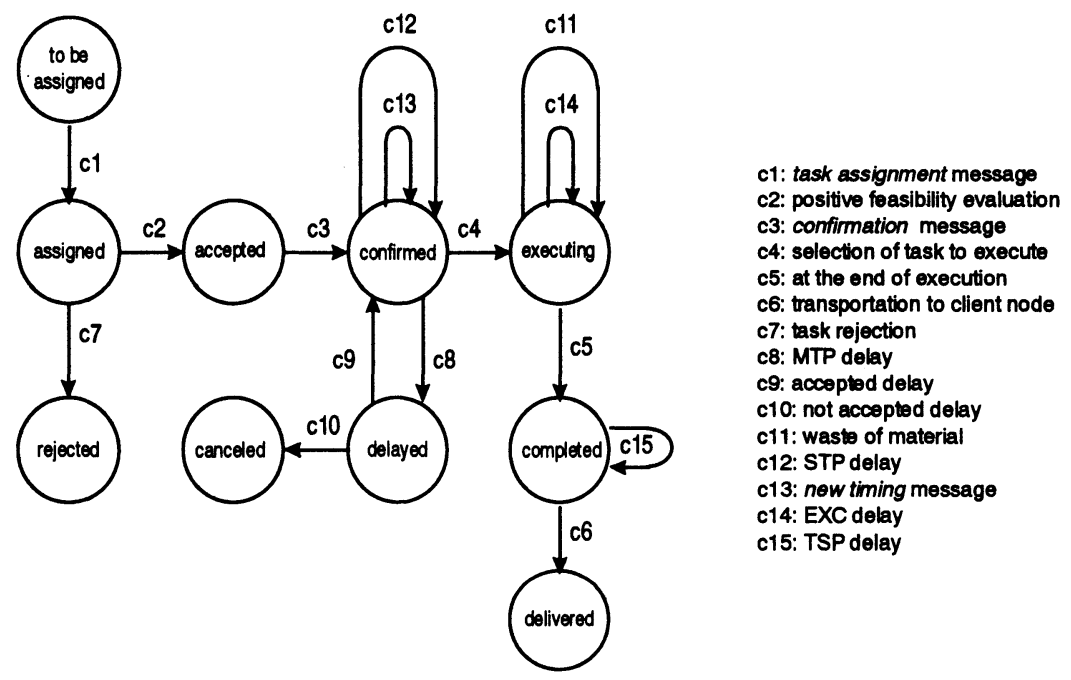

Figure 2 State diagram of the single task.

In its turn, every tasks assigned to a node is represented by the triplet $\langle p, q, d d\rangle$ (phase type, quantity, due date). Its evolution occurs according to the rules represented in Figure 2 . At the end of the assignment process, the task (whose value is assigned) is communicated to the involved node which verifies (manually or automatically) its feasibility. As soon as this, and all the other tasks resulting from a customer order, is accepted by the relative node, the order changes its state from suspended to confirmed, and the tasks itself is confirmed by the coordinating unit back to node of interest.

Starting from this situation, the node will inform the $\mathrm{CU}$ on task evolution whose correct sequence of state values includes executing, completed and delivered. Instead, if the tentatively assigned task is rejected, its state changes from assigned to rejected (and, therefore, the order to which it belongs is replanned).

A confirmed task is planned for execution by the involved node. If a delay is detected (c8) the task state becomes delayed until a decision is taken by the coordinating unit (either the 
delay is accepted or the task assignment is cancelled). Another task replanning may occur if the delay is detected at the scheduling time. The consequence of replanning is that sometimes the $\mathrm{CU}$ must communicate to nodes new timings of already confirmed tasks.

\section{NODE PROCESSES}

With the PLENT approach nodes are expected to maintain a high autonomy of local planning, on the medium and short term horizons, and of task execution. They are required to interact with the CU in order to make this aware of any significant state change of the assigned tasks and, more important, of any possible delay or material waste that can affect the order progress. $\mathrm{CU}$ is in charge of managing all synchronization problems the single node cannot face autonomously. This satisfies a specific requirement coming from real cases, where the burden of frequent and informal communications between nodes is considered one of the major drawbacks of distributed manufacturing with respect to centralized organization.

Network behaviour is described by a list of conditions which activate certain processes, those which take place inside the nodes or the coordinating unit. The triggering condition associated with a process is constituted by:

- a message coming from an external entity (e. g. a communication about a task assignment between the coordinating unit and a certain node or an order deletion directly decided by a customer),

- an event that occurs inside the examined entity (e. g. a delay during manufacturing inside a node or the expiring of the scheduled time between two medium term plannings).

As result, each process can involve in its turn external entities sending them new messages. The main functions performed by a node are represented in the data flow diagram of Figure 3.

\section{ACC: acceptance/rejection of an assigned task}

This process is executed whenever a task assignment message comes from the CU. Since CU carefully considers the node declared availability and previous assignments, task acceptance is the expected answer (the task is recorded as accepted but not yet confirmed). Task rejection will make node position in the network worse (the task is recorded in the task log file).

The decision about acceptance/rejection of the task, on behalf of the node, can be automatically reached (e. g. by means of a medium-term planning simulation) or according to heuristic criteria: what really results critical is the node rapidity to send the answer to the CU. This, after having calculated tasks distribution among $\boldsymbol{n}$ nodes to carry out a certain order, waits for the confirmation of all the nodes involved. Only after the $n$ acceptance messages have been received by the $\mathrm{CU}$, this sends a confirmation signal.

Usually, node answer will be positive, hence the ACC will result in updating the data structure storing the related tasks. At the end of the process, the node waits for the $\mathrm{CU}$ confirmation signal.

\section{CFM: accepted task confirmation}

The triggering condition of the CFM process is the confirmation/rejection signal simultaneously sent to all the nodes involved in the tasks distribution. If all the assigned tasks 
have been accepted, then the CU sends to them a confirmation signal. Instead, if a node has rejected its duty, it is necessary that the CU performs a new planning.

If we suppose that $\boldsymbol{m}$ of the $\boldsymbol{n}$ tasks have been rejected (a quite improbable event) then the CU can manage this situation in two ways: (i) by assigning the $m$ tasks to other nodes, that should confirm in their turn without modifying the distribution involving the $n-m$ tasks already assigned; (ii) by calculating a new task distribution involving also some of the $n-m$ tasks already assigned; (iii) by failing in searching new assignments which are able to satisfy customer requests, in which case it is necessary to negotiate a new due date with customer (moreover, all the tasks are deleted and afterwards are assigned again according to a new distribution). In the last two cases, the CU can deny a task assignment even if it was already accepted by a node. Therefore, the examined task is removed from the set of those accepted.

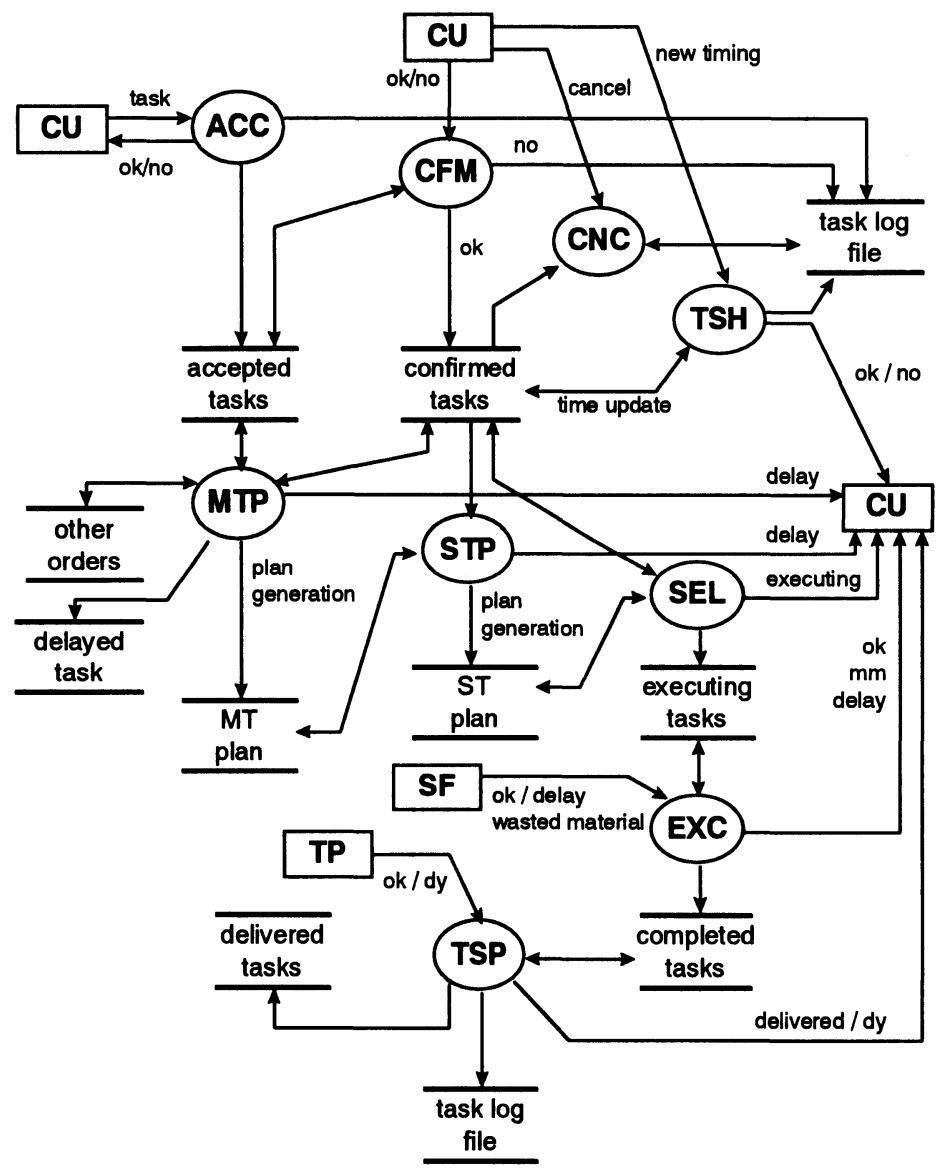

Figure 3 Diagram of the node processes. 


\section{MTP: medium term planning}

Unlike the two activities seen above, whose activation depends on signals coming from the external enviroment, the MTP process is cyclically performed (e. g. two times a week) in order to provide forecasts regarding tasks execution and information about purchase orders.

The process is applied to all the orders received by a node, from the CU or from other customers, directly. This process takes into account either the confirmed tasks or the accepted ones which have not yet been confirmed. The process results in a medium term planning where each order or task refers to the temporal axis which determines the beginning of the working. The accuracy of the start and end times strongly depends on the characteristics of the MTP package, which can differ according to the node examined.

At the end of the planning process some tasks can change their state according to their finishing dates as they are determined by MTP. In particular, if the finishing date preceedes the due date, no state change occurs, otherwise some perturbations involving network behaviour can raise. Whenever a node is not able to carry out a certain task within the period fixed by the coordinating unit, the involved task is marked as delayed and a message is sent to the CU. Then, the CU will try to distribute these phases among the nodes following the examined one, by means of partial replanning.

\section{STP: short term planning}

Similarly to the MTP process, also this process is executed cyclically (usually, once a day) in order to schedule the production orders that refer to the next hours. The STP process only takes into account tasks whose state assumes the confirmed value and whose start date (that fixed by the MTP process) falls within the temporal horizon considered by short-term planning.

The result of the STP process is a plan where the examined tasks are placed on the temporal axis showing their execution sequence. Besides, the level of detail reached during STP is higher than the one corresponding to MTP since the start and end dates considered by the latter are only foreseen but not definitively fixed.

The STP process allows the node to know whether its tasks can be carried out within the date fixed by the CU. Shall this condition not occur, the corresponding signal has to be sent by the examined node to the coordinating unit. The $\mathrm{CU}$, in its turn, has to warn the nodes following the examined one in the network, against the perurbations determined by the delay that affects the materials delivery.

\section{SEL: selection of the task to be executed}

It is the aim of this process to select the tasks to be executed: hence, their state passes from the confirmed value to the executing one. The coordinating unit is informed on tasks state change.

\section{EXC: control of tasks to execute}

Whenever a task assigned to a node is completely accomplished, without determinimg perturbations, a positive acknowledgment is sent from the shop-floor. This message triggers another one, sent by the node to the coordinating unit, showing that the new value assumed by the task state is the executed one.

Nevertheless it could happen that a task is not carried out within the time that has been previously fixed due to materials acquisition or working problems. In this case, the node has to inform the $\mathrm{CU}$ on the problems occurred (by means of messages like delay or missing 
materials). The $\mathrm{CU}$ will try to overcome the drawbacks by assigning some tasks to other nodes or negotiating new requests with the customer.

\section{TSP: transportation to the next node (or to the customer)}

Every time a task is completely executed, its product is delivered to the entity responsible for the transportation to the next node (or to the customer). After this activity execution, the task state becomes delivered.

\section{CNC: task cancellation}

Whenever the coordinating unit deletes a task that have already been assigned (and accepted) to a node, because of some problems such as those seen above, the CNC process of the examined node has, in its turn, to delete the task from the accepted ones.

\section{TSH: task timing shifted}

Every time a node informs the $\mathrm{CU}$ on a possible delay, the coordinating unit tries to calculate a new task distribution involving the tasks assigned to the node considered and causing the delay. Hence, it is necessary a partial re-planning that takes into account only the nodes that have not yet executed their tasks so as to be able to move up the date that refers to the production order release. Therefore, the TSH process takes place in these nodes. Other nodes that are not able to update the production order release are only informed by the CU on possible perturbations.

\section{COORDINATING UNIT PROCESSES}

The main functions carried out by the coordinating unit arerepresented in the data flow diagram of Figure 4. Processes ASS, RRP, MDLY, SDLY are all related to workload distribution to the nodes, based on the following principles:

- One order at a time. Splitting customer orders into node tasks is performed order by order. Since the external request should be answered in the shortest time, the CU cannot cumulate it with other orders for optimization purposes, but must process it immediately.

- Task assignment at the latest. Assignment of order tasks to nodes is carried out according to a backward approach, taking into account the tasks already distributed to nodes. Thus, the network nodes receive the communication of their involvement with the widest time margins and can plan the respective activities in the best conditions.

- Balanced node involvement. The network nodes have equal rights, hence the assigned workloads are proportional to the respective available capacities, considering also the already assigned workloads and the node reliability. The daily available capacity of a node with respect to a given phase is that declared by the node itself (possibly corrected, as we shall see), decreased to take into account previous assignments.

- Node internal concurrency. The resources of a node can be used to perform operational phases in alternative; it means that assignment of a workload for a given phase subtracts resources to the execution of the other phases. In order to take into account these situations, whenever a task is charged onto a node the capacity of the relative phase is 
decreased, together with a proportional decrement of the capacities of all the concurrent phases.

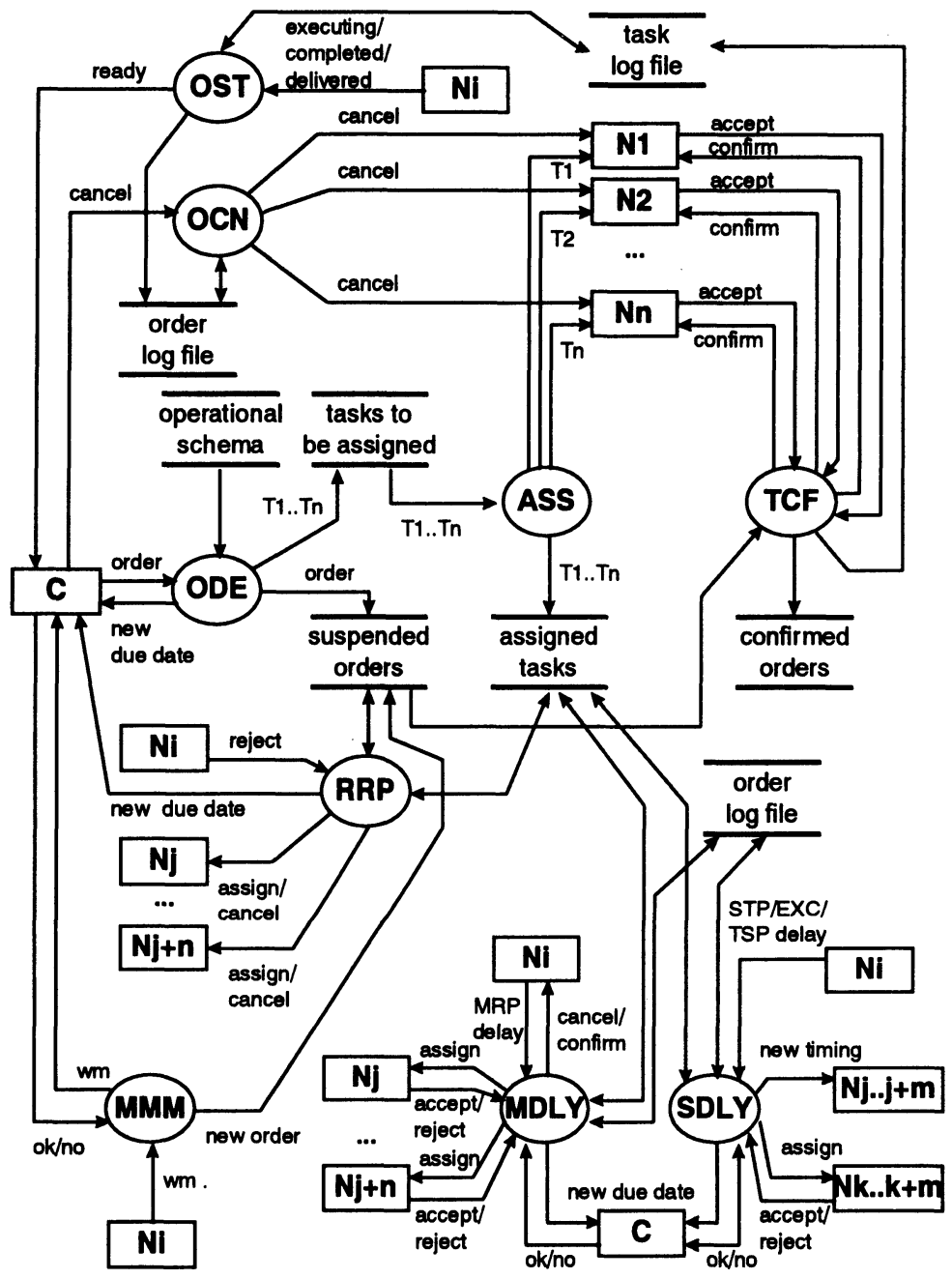

Figure 4 Diagram of the coordinating unit processes.

Some particular events could require new partial or global planning in charge of the coordinating unit. In particular, new replanning is necessary every time that the network receives a new order, or a task is rejected by a node, or the MTP process of a node points out a delay, or the EXC process of a node points out wasted materials. 
Instead, if the delay is due to STP, EXC or TSP, the coordinating unit only informs the corresponding nodes on the time shifting that affects the material due date.

\section{ODE: customer order decomposition into tasks}

The ODE process is executed whenever the coordinating unit receives an order from a customer. It is the aim of this process to apply a proper algorithm that calculates a tasks distribution according to the information related to the network operational schema. The new order assumes the suspended state until the corresponding tasks are accepted by the nodes.

\section{ASS: tasks assignment to network nodes}

This process starts after the proper workload distribution has been calculated by the above algorithm. During this phase the coordinating unit informs all the nodes, involved by the workload distribution, on the tasks assigned to them. Obviously, more tasks can be assigned to the same node.

\section{TCF: tasks confirmation to the nodes and the customer}

After task distribution, the coordinating unit waits for the answers from the nodes involved in the distribution itself. It is clear that the answers have to reach the $\mathrm{CU}$ within a certain time, usually fixed by the network management. If all the nodes send to the CU a message whose meaning is task accepted, the $\mathrm{CU}$, in its turn, can start the confirmation process that definitely assigns the tasks to the nodes, as in a two phase commit communication protocol.

The order corresponding to the examined tasks changes its state from the suspended to the confirmed values. Afterwards, a confirmation signal is sent to the customer responsible for the order.

\section{$R R P$ : replanning due to task rejection}

If one (or more) of the above nodes rejects the tasks assigned by the CU, it is necessary a new assignment process in order to replace this node (these nodes). The new planning phase could determine a new tasks assignment involving also the tasks already assigned, or another negotiation with the customer.

Obviously, at the end of the re-planning phase it is necessary to perform the TCF process again. Moreover, it could be impossible to find out a new distribution tasks after rejection: in this case, the coordinating unit has to negotiate with the customer another due date.

\section{OST: order and tasks state monitoring}

This process allows the coordinating unit to update the task and the order state using the information associated to the messages coming from the nodes. As soon as the enterprise in charge of the operational schema root communicates that its duty has been accomplished, the CU informs the customer on product delivery.

\section{OCN: order cancellation}

Whenever some problems are detected, the coordinating unit has to inform the customer on the need of fixing a new due date.

If the customer decides to delete the order, the OCN process provides the coordinating unit to inform the nodes involved in the tasks distribution on the deletion of their tasks; moreover, the order and these tasks assume the cancelled state. 


\section{MDLY: replanning due to MTP delay}

This process is executed by the coordinating unit every time problems occur during the medium-term planning performed by a single node. In this case the node communicates to the coordinating unit the delay its MTP has foreseen and the CU starts a new planning phase that is managed by the MDLY process.

During this replanning phase, redistribution of the tasks that follow the delayed ones, can involve all the nodes following the one that has detected the problem. The result of the process is a new distribution involving all the tasks that follow the one that has determined the delay. Moreover it is possible that the CU assigns this task to another node. After having obtained the new distribution, all the nodes considered by the new distribution have to send to the $\mathrm{CU}$, within a fixed time interval, a confirmation message: this message is received by means of the TCF process.

The tasks that have been previously assigned to the nodes following the one responsible for the delay cannot be removed, since this delay do not depend on these nodes. Therefore the CU can only require an effort to them in order to anticipate the due date that refers to their products: this kind of effort will be payed by assigning a positive score to the examined nodes.

If the coordinating unit is not able to find out a new proper distribution it is necessary a new negotiation phase with the customer.

\section{SDLY: management of delays due to STP, EXC, TSP}

This process (similar to that above) is performed by the coordinating unit as reaction to the messages coming from the nodes that have detected problems during short-term planning, task execution or transfer phase.

Since the delay can be detected when the task is almost accomplished, the CU cannot remove the phase from the node duty or move up all the tasks that have to be executed by the following nodes. Instead, the coordinating unit has to identify the two following sets of nodes: the set of node whose tasks are still managed by the medium-term planning; the set of nodes whose tasks are already taken into account by the short-term planning or are running.

As result of a re-planning phase all the nodes belonging to the first set will be required to anticipate their task ends (this is performed by means of assign, accept, confirm messages) so as to balance the delay due to the nodes of the second set. As to the nodes belonging to the second set, they are informed (by means of a new timing message) on their tasks moving on the time axis.

If some nodes cannot accept the results of the re-planning phase it is necessary (just as in the above process) to inform the customer on the delay.

\section{MMM: material wastes management}

This process is executed whenever a material waste message is sent to the CU by a node. It is the aim of this process to decrease the quantity of the ordered product provided that the customer is satisfied. Otherwise, it is necessary to create a new order that is able to balance the wasted material. 


\section{CONCLUSIONS}

The control architecture adopted for the activities carried out in a network of small-medium manufacturing enterprises has been presented in terms of information flows and processes involving the network basic entities, namely customer, coordinating unit and nodes. This solution has been identified to meet two fundamental aims which take into account the peculiar nature of SMEs:

- Autonomy. Every network node is an enterprise used to operate alone on a market populated of many competitors, often located in the same industrial district. This results in a strong spirit of independence that might interfere with the participation in the network. Only an organizational paradigm which leaves the single node free to decide its role in the network, ensuring at the same time the correct application of the agreed management rules by a neutral coordinating unit, can overcome distrust and possible difficulties.

- Sustainability. The new organizational paradigm should not require significant modifications of the node organization. The node must remain free to participate in different networks and also maintain its direct customers. The benefits ensured by the virtual enterprise (wider market, incentive to improve productivity and quality) shall not be payed with a loss of flexibility and initiative. As soon as further organizational models will be identified, the single enterprise must still be in condition to try them.

A validation activity of the proposed model has been carried out on three european networks (in Italy, Spain and Hungary) in order to test the model applicability, verify the planning paradigm principles and receive indication from end users about model enhancement and correction. The study cases range from small batch production of oleodynamic components to one-of-a-kind production of transfer machine tools for the automative industry.

At present, the PLENT project is completing the software design phase, and the development phase is going to start.

\section{REFERENCES}

Bonfatti F., Monari P.D. (1995) Planning small-medium enterprise networks. International Conference on Improving Manufacturing Performance in a Distributed Enterprise (IMPDE '95), Edinburgh.

Bonfatti F., Monari P.D., Paganelli P. (1996) Coordination function in SME networks. International Conference on Architectures and Design Methods for Balanced Automation Systems (BASYS '96), Lisbon.

Koestler A.(1989) The Ghost in The Machine. Arkana Books.

Rolstadas A. (1994) Beyond year 2000 - production management in the virtual company. IFIP Transactions B-19 on Production Management Methods, North Holland.

Hirsch B. E. et al. (1995) Decentralized and collaborative production management in distributed manufacturing environments, in Sharing CIME Solutions (eds. J. H. K. Knudsen et al.), Ios Press.

PLENT Consortium (1996) Network Policy Modelling. Deliverable D01.

PLENT Consortium (1997) Software Design. Deliverable D02. 